\author{
Małgorzata Ostrowska ${ }^{1 *}$, Piotr Adamski ${ }^{1 *}$, Marek Koziński ${ }^{1}$, Eliano Pio Navarese ${ }^{2}$, Jacek Kubica ${ }^{3}$ \\ ${ }^{1}$ Department of Principles of Clinical Medicine, Collegium Medicum, Nicolaus Copernicus University, Bydgoszcz, Poland \\ 2Department of Internal Medicine, Division of Cardiology, Pulmonology and Vascular Medicine, Heinrich-Heine-University, Düsseldorf, Germany \\ ${ }^{3}$ Department of Cardiology and Internal Medicine, Collegium Medicum, Nicolaus Copernicus University, Bydgoszcz, Poland \\ *Matgorzata Ostrowska and Piotr Adamski have equally contributed to the present paper and should be considered first authors
}

\title{
Diabetogenic effect of statins: a comprehensive review on the clinical relevance, underlying pathomechanisms and rationale for tailored statin therapy
}

\author{
Corresponding author: \\ Małgorzata Ostrowska, MD, PhD \\ Department of Principles of Clinical \\ Medicine \\ Collegium Medicum, Nicolaus \\ Copernicus University \\ 9 Skłodowskiej-Curie Street \\ 85-094 Bydgoszcz, Poland \\ Tel.: +48525854023 \\ Fax: +48525854024 \\ E-mail: ostrowska.go@gmail.com
}

\begin{abstract}
Statins are potent hypolipidemic drugs effectively reducing low-density lipoprotein (LDL) cholesterol serum concentration, but also exerting a wide range of pleiotropic effects. In numerous clinical trials statins were proven to substantially decrease cardiovascular morbidity and mortality both in primary and secondary prevention. However, a growing body of evidence suggests that statins, although safe and generally well-tolerated, are associated with an increased occurrence of new-onset diabetes mellitus (DM). The aim of this review is to explore the relationship between statin therapy and new-onset DM, including its clinical relevance and underlying pathomechanisms, and to discuss the concept of tailored statin therapy. According to our recently published comprehensive network meta-analysis including 113,394 patients, the high-dose statin regimens were connected with an elevated risk of new-onset DM as compared with moderate-dose statin regimens and a gradient for the risk of new-onset DM across different types and doses of statins was demonstrated. There are multiple possible mechanisms explaining the diabetogenic effect of statins (e.g., decreased insulin secretion, induction of $\beta$-cell apoptosis, increased insulin resistance or compromised glucose transport into the cells). Statins are among the most widely used drugs worldwide and physicians should be aware of the fact that there is a risk of new-onset DM across different types and doses of statins. Selection of adequate statin that suits patient's needs remains the challenge of hypolipidemic therapy. The identification of individuals who would benefit more from smaller doses and/or use of less diabetogenic compounds could help to optimize the treatment and reduce the number of patients developing DM. The non-pharmacological approach such as adequate physical activity, weight reduction and low fat diet should not be neglected either. These actions create a chance to decrease baseline LDL-cholesterol concentration and reduce the number of both cardiovascular and DM risk factors. All in all, statins with their exceptional cardiovascular benefits will undoubtedly defend their position of a cornerstone of cardiovascular prevention because profits derived from statin therapy far exceed the potential harms connected with statin-induced impairments of glucose metabolism.

Key words: diabetes, diabetogenicity, statins, hydroxy-methylglutaryl coenzyme A (HMG-CoA) reductase inhibitors, adverse effects, personalized statin therapy
\end{abstract}

Folia Medica Copernicana 2015; 3 (4): 145-153
Folia Medica Copernicana 2015 Volume 3, Number 4, 145-153 10.5603/FMC.2015.0009 Copyright @ 2015 Via Medica ISSN 2300-5432

\section{Introduction}

Statins are potent hypolipidemic drugs which selectively and reversibly inhibit the enzyme 3-hydroxy-methylglutaryl coenzyme A (HMG-CoA) reductase. These agents suppress cholesterol synthesis in the liver and increase expression of low-density lipoprotein (LDL) receptors in hepatocytes leading to enhanced LDL-cholesterol uptake. Both actions finally result in a reduced concentration of LDL-cholesterol in the bloodstream [1-4]. 
Apart from the influence on LDL-cholesterol concentration, statins also exert a wide range of pleiotropic effects, including anti-inflammatory and anti-oxidative properties, stabilization of atherosclerotic plaques, decreased blood thrombogenicity, and improvement of endothelial function [5-8]. Most importantly, in numerous clinical trials statins were proven to substantially decrease cardiovascular morbidity and mortality both in primary and secondary prevention [9-25]. Based on the observation that even a modest impact of statins on LDL-cholesterol concentration and atherosclerotic plaque burden is associated with the substantially reduced rate of cardiovascular events, the above listed pleiotropic effects are suggested to be at least partially responsible for clinical benefits of statin therapy.

Since the first group representative, lovastatin, was introduced to the market in 1987, statins have become one of the most frequently prescribed drugs worldwide. A growing body of evidence suggests that statins, although safe and generally well-tolerated, are associated with several adverse events, with the increased occurrence of new-onset diabetes mellitus (DM) being the most widely discussed in recent years [26-29]. These findings have even forced the US Food and Drug Administration to add information to statin labels about the increased risk of raised blood glucose concentration and development of type 2 DM [30].

Our recently published meta-analysis constitutes the largest and the most comprehensive so far conducted evaluation of the risk of new-onset DM associated with different types and doses of statins [31]. We believe that the findings of this meta-analysis, if properly applied in clinical practice, could help personalize statin therapy and confine the adverse diabetogenic effect of statins in specific patient subsets.

The present review aims to explore the relationship between statin therapy and new-onset DM, including its clinical relevance and underlying pathomechanisms, and to discuss the concept of tailored statin therapy.

\section{Search strategy}

A search covering the period from November 1994 through October 2015 was conducted by two independent investigators using MEDLINE, CENTRAL and Google Scholar databases. Proceedings from the Scientific Sessions of the American College of Cardiology [http://www.acc.org], American Heart Association [http://www.heart.org], the European Society of Cardiology [http://www.escardio.org], Transcatheter Cardiovascular Therapeutics [http://www.tctmd.com] and EuroPCR [http://www.europcr.com] were also considered. The following keywords were applied: "diabetes", "diabetogenicity", "glucose", "statin", "hydroxy-methyl- glutaryl coenzyme A (HMG-CoA) reductase inhibitors", "adverse effect". References of the retrieved studies were searched manually for additional studies and reviews. No language restrictions were applied.

\section{Overview of studies linking statin therapy with new-onset DM}

The first report of possible connection between statin therapy and the development of new-onset DM was published in 2001 by the authors of a post hoc analysis of the WOSCOPS (West of Scotland Coronary Prevention Study) trial, a randomized placebo-controlled study including 5974 men with hypercholesterolemia and no history of myocardial infarction [11]. Unexpectedly, the study indicated a $30 \%$ reduction in the incidence of DM among pravastatin- vs. placebo-treated patients (hazard ratio [HR] 0.70; 95\% confidence interval [Cl] Cl 0.50-0.99). However, it needs to be acknowledged that the authors used non-standardized criteria for the DM diagnosis.

This protective effect of statins on new-onset DM occurrence was not confirmed in subsequent clinical studies. On the contrary, the results of the JUPITER (Justification for the Use of Statins in Primary Prevention: an Intervention Trial Evaluating Rosuvastatin) trial indicated quite the opposite [12]. Despite the fact that in 17,802 apparently healthy men 50 years of age or older, and women 60 years of age or older, with LDL-cholesterol concentration of less than $130 \mathrm{mg} / \mathrm{dL}$ and C-reactive protein concentrations of $2.0 \mathrm{mg} / \mathrm{L}$ or higher, rosuvastatin 20 mg q.s., as compared with placebo, was proven to significantly reduce the primary study endpoint, a composite of myocardial infarction, stroke, unstable angina, necessity of arterial revascularization procedure and death from cardiovascular causes, it simultaneously raised the rate of newly diagnosed DM and increased the median value of glycated hemoglobin.

Numerous other randomized clinical trials, whose both design and major findings are summarized in Table 1, also suggested excess of new-onset DM in statinv. placebo-treated patients.

Furthermore, Rajpathak et al. in a meta-analysis of five randomized statin trials reported an overall risk ratio (RR) of 1.13 with a $95 \% \mathrm{Cl}$ ranging from 1.03 to 1.23 for new-onset DM in statin-treated patients as compared with the placebo/no treatment group [26]. However, when the data from the WOSCOPS trial was included in the meta-analysis, the RR value decreased to 1.06 and was no longer statistically significant (95\% Cl 0.93-1.25).

Another substantial argument supporting the diabetogenic effect of statins was delivered by Sattar and colleagues, who performed a meta-analysis of thirteen randomized statin studies including 91,140 participants [28]. 
During a mean follow-up of 4 years, 4278 patients developed DM, of whom 2226 and 2052 were in the statin and control groups, respectively. In this meta-analysis, statin therapy was associated with $9 \%$ higher risk of developing new-onset DM as compared with the control arm (OR 1.09; 95\% Cl 1.02-1.17). In other words, treatment of 255 patients with statins for period of 4 years resulted in one additional case of DM. Interestingly, meta-regression analysis showed that neither LDL-cholesterol concentration nor baseline body-mass index had any impact on the risk of DM development, whereas elderly age was associated with an increased occurrence of new-onset DM.

Likewise, the dosage of statins appears to be directly connected with their diabetogenicity. Preiss et al. investigated this hypothesis in a meta-analysis of five randomized statin trials including 32,752 participants without DM at baseline who were either assigned to high- or moderate-dose therapy [29]. Out of 2749 patients who developed DM during the follow-up, 1449 and 1300 were treated with high- and moderate-dose statin therapy, respectively. The patients subjected to high-dose statin therapy, compared with participants on moderate-dose statin therapy, were more likely to develop DM (odds ratio [OR] 1.12; 95\% Cl 1.04-1.22), but at the same time suffered less major adverse cardiovascular events (OR $0.84 ; 95 \% \mathrm{Cl} 0.75-0.94$ ). The corresponding number-needed to treat (NNT) and number-needed to harm $(\mathrm{NNH})$ values were 155 and 498 , respectively.

\section{Recent network meta-analysis of impact of different types and doses of statins on new-onset DM}

The outcomes of the aforementioned trials and analyses raised many concerns in the medical world and left us with unanswered questions of how to use statins in order to minimize the potential risk of generating new-onset DM and how to optimize their beneficial effects in cardiovascular prevention at the same time. These unsolved issues led us to conduct a comprehensive network meta-analysis, in which we compared the impact of different types and doses of statins on new-onset DM [31]. The data acquired from seventeen randomized controlled trials, covering 113,394 patients, were subjected to the investigation. Fourteen trials compared statin with placebo/no treatment and three studies compared high-dose (atorvastatin $80 \mathrm{mg}$ q.s., lovastatin 20-40 mg q.s., pravastatin $40 \mathrm{mg}$ q.s., rosuvastatin $20 \mathrm{mg}$ q.s. or simvastatin $40 \mathrm{mg}$ q.s.) with moderate-dose therapy (atorvastatin $10 \mathrm{mg}$ q.s., pravastatin 10-20 mg q.s. or rosuvastatin $10 \mathrm{mg}$ q.s.). Noteworthy, treatment with rosuvastatin $20 \mathrm{mg}$ q.s. was found to increase the relative risk of DM incidence by $25 \%$ compared with the placebo group, with a similar magnitude of increased risk observed in patients treated with simvastatin $40 \mathrm{mg}$ q.s. Rosuvastatin was also associated with the highest risk of new-onset DM. On the other hand, pravastatin, both in high- and moderate-doses, exposed meta-analysis participants at the lowest risk of DM as compared with placebo. Moreover, the risk for DM in subjects treated with pravastatin $40 \mathrm{mg}$ q.s. did not differ very much from the risk observed in patients from the placebo group. Superior safety profile, in terms of the new-onset DM occurrence, of pravastatin over rosuvastatin was also observed when these compounds were compared directly and a $16 \%$ relative risk reduction of the incidence of new-onset DM was reported in favor of pravastatin. Similarly, therapy with atorvastatin $80 \mathrm{mg}$ q.s. resulted in approximately $8 \%$ relative risk reduction for new-onset DM compared with high-dose rosuvastatin. At moderate doses the risk of developing DM while using atorvastatin or rosuvastatin was comparable. In general, high-dose statin regimens were connected with elevated risk of new-onset DM as compared with moderate-dose statin regimens and a gradient for the risk of new-onset DM across different types and doses of statins was demonstrated.

\section{Potential mechanisms underlying the diabetogenic effect of statins}

There are multiple possible mechanisms which could serve as an explanation of the observed association between statin therapy and new-onset DM (Fig. 1). Statins

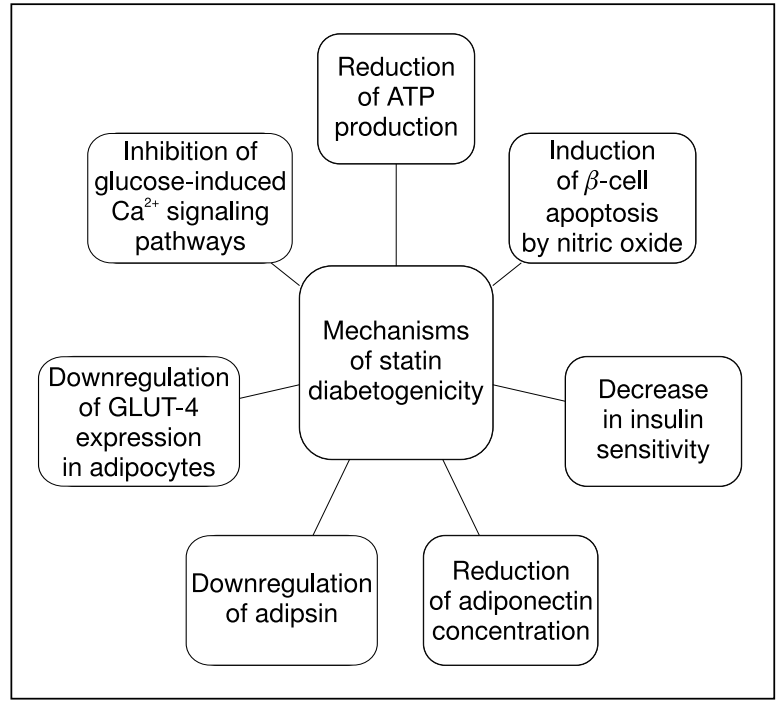

Figure 1. Proposed mechanisms of statin diabetogenicity; ATP — adenosine triphosphate; GLUT — glucose transporter 


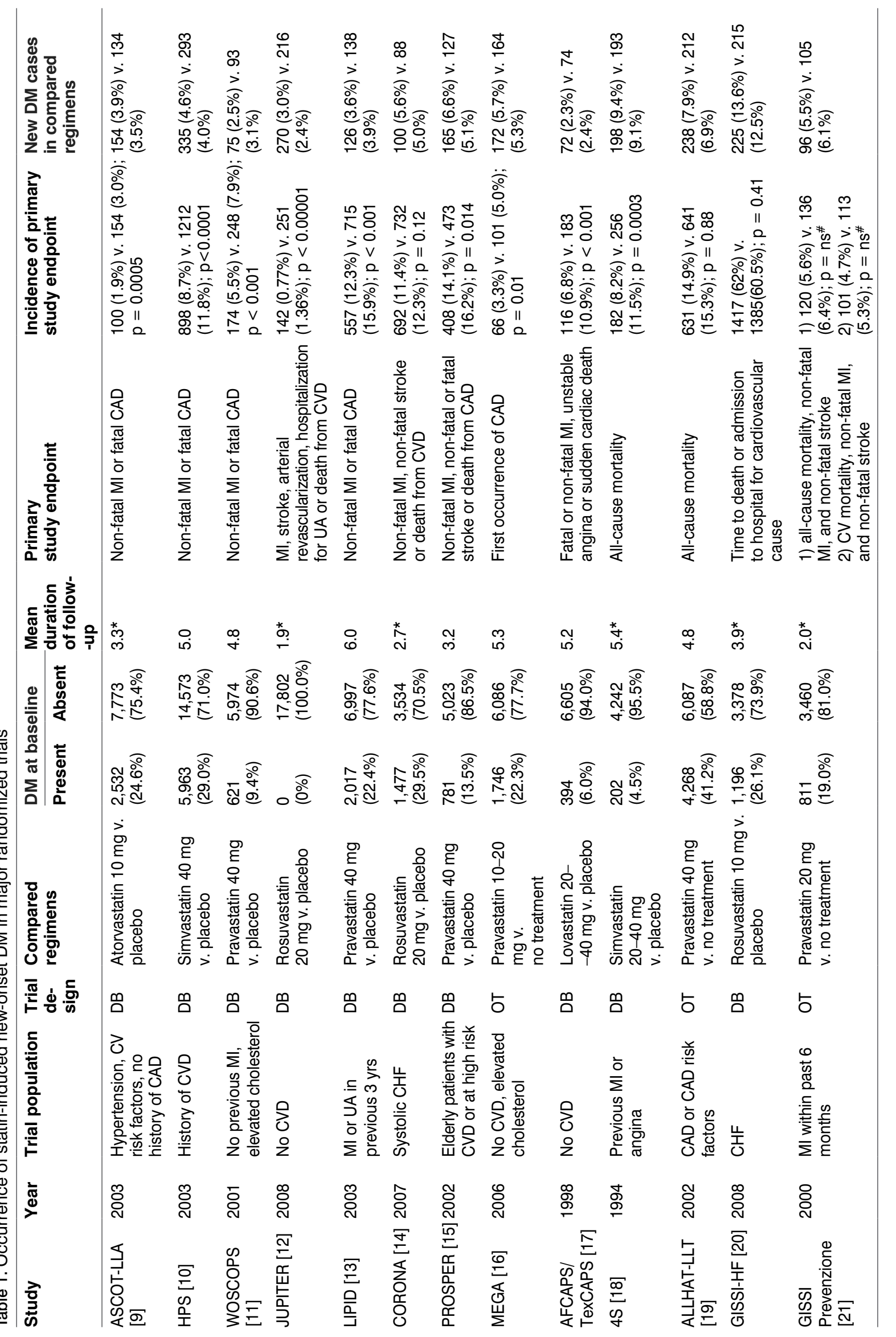




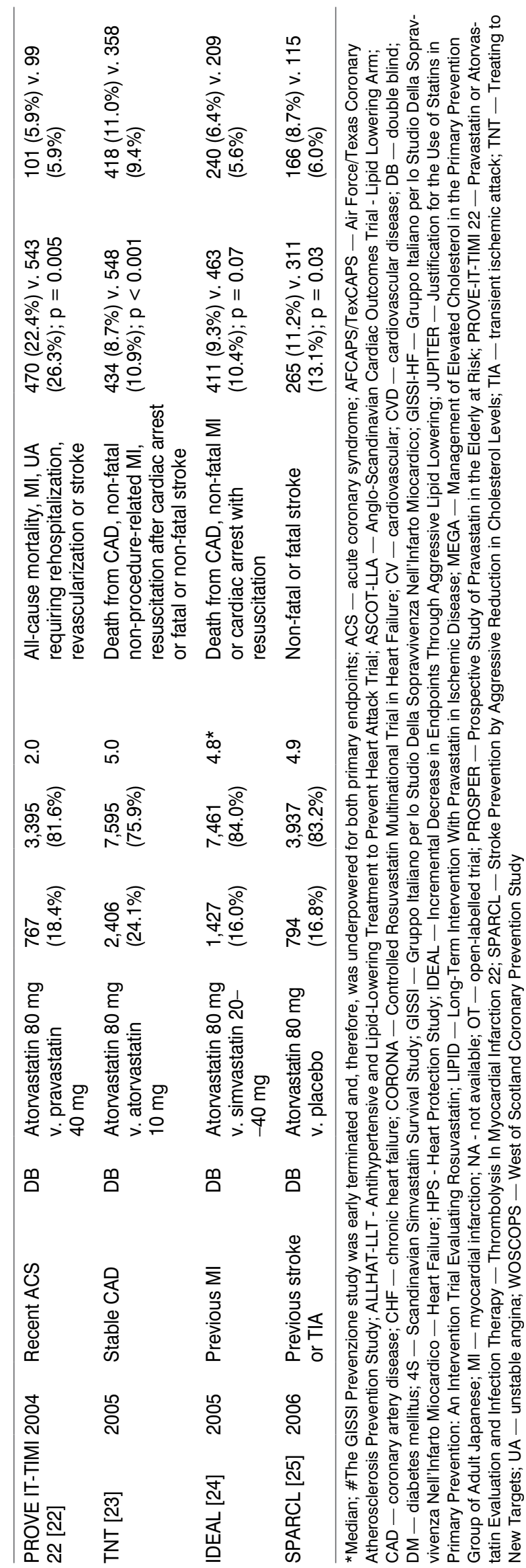

may influence the pancreatic $\beta$-cell function and insulin secretion via inhibition of glucose-induced $\mathrm{Ca}^{2+}$ signaling pathways. Simvastatin, but not pravastatin, was also found to block the L-type $\mathrm{Ca}^{2+}$ channels, inhibiting insulin secretion in rat islet $\beta$-cells [32]. Additionally, insulin release may also be impaired by decreased amount of adenosine triphosphate. Of note, statins suppress the ubiquinone biosynthesis, which results in delayed and reduced production of adenosine triphosphate [33]. Another postulated mechanism of statin diabetogenicity is the induction of $\beta$-cell apoptosis by nitric oxide [34].

As demonstrated in our meta-analysis and in other studies, statins do not exert a class effect on the new-onset DM occurrence and insulin sensitivity, however, substantial intra-class differences have been observed. According to a study by Baker et al., pravastatin significantly improves insulin sensitivity as compared with placebo, while simvastatin worsens it [35]. Simvastatin was reported to significantly reduce concentration of adiponectin, a protein hormone attenuating gluconeogenesis and stimulating glucose uptake, and insulin sensitivity in hypercholesterolemic patients [36, 37]. It is hypothesized that lipophilic and hydrophilic statins have different effects on adiponectin and insulin resistance. Pravastatin, a representative of hydrophilic statins, increases adiponectin concentration and insulin sensitivity. In contrast, simvastatin was reported to significantly increase fasting insulin concentration, worsen insulin resistance and reduce adiponectin concentration [38]. Bhandari et al. postulated a possible link between statin therapy and downregulation of adipsin, a novel adipokine responsible for the stimulation of insulin secretion by pancreatic $\beta$-cells, resulting in an increased risk of new-onset DM [39]. Notably, glucose is transported into the cells via insulin-regulated transporters. One of them, glucose transporter type 4 (GLUT-4) is responsible for glucose entrance to adipocytes and skeletal muscle cells. Atorvastatin was demonstrated to downregulate the expression of GLUT-4 in adipocytes, thus impairing glucose tolerance [40]. According to a hypothesis formulated by Chamberlain, statin-induced attenuation of GLUT-4 expression in adipocytes may be explained by inhibition of the isoprenoid synthesis [41]. Swerdlow et al. proved that glucose-rising effect of statins may be at least partially explained by HMG-CoA reductase inhibition [42]. They used single nucleotide polymorphisms (rs17238484-G and rs12916 alleles) in the enzyme HMG-CoA reductase gene as surrogates for HMG-CoA reductase inhibition by statins in a group of 223,463 patients. Having analyzed these genetic variants and a combination of their own and published data, the authors demonstrated that the presence of both investigated allelic variants was not only associated with a decreased HMG-CoA reductase activity, but also with a higher risk of statin-induced type $2 \mathrm{DM}$ 
(the rs17238484-G allele: OR 1.02; 95\% Cl 1.00-1.05; the rs12916 allele: OR 1.06; 95\% Cl 1.03-1.09).

Although the above proposed mechanisms could contribute to the diabetogenic effect of statins, a definitive mechanism of this phenomenon has not been clarified so far.

\section{Statin therapy in patients with diagnosed DM}

It is important to emphasize that in patients with previously diagnosed DM statin therapy is a mainstay of treatment and brings substantial cardiovascular benefits. This was clearly established by the Cholesterol Treatment Trialists' (CTT) Collaborators in a meta-analysis of fourteen randomized trials including 18,686 patients with DM (1466 with type $1 \mathrm{DM}$ and 17220 with type 2 DM) and 71,370 non-diabetic patients [43]. Statin therapy, when compared with placebo/no treatment, led to a $9 \%$ decrease of all-cause mortality per $1 \mathrm{mmol} / \mathrm{L}$ reduction of LDL-cholesterol in participants with DM (RR 0.91; 99\% Cl 0.82-1.01), which was comparable to the $13 \%$ reduction in those without DM (RR $0.87 ; 99 \%$ $\mathrm{Cl}$ 0.82-0.92). The mortality benefits in diabetics were mainly driven by reduced vascular mortality (RR 0.87 ; $95 \% \mathrm{Cl} 0.76-1.00)$. There was also a $21 \%$ reduction in major vascular events per $1 \mathrm{mmol} / \mathrm{L}$ decrease of LDL-cholesterol concentration in DM patients (RR 0.79; 99\% Cl 0.72-0.86), similarly to non-diabetics (RR 0.79; $99 \% \mathrm{Cl} 0.76-0.82)$. Substantial reductions in myocardial infarction or coronary death (RR $0.78 ; 99 \% \mathrm{Cl} 0.69-0.87$ ), coronary revascularization (RR $0.75 ; 99 \% \mathrm{Cl} 0.64-0.88$ ), and stroke (RR $0.79 ; 99 \% \mathrm{Cl} 0.67-0.93$ ) were observed in statin vs. placebo-treated diabetics. It should be acknowledged that larger reductions in LDL-cholesterol concentration produced greater reductions in the occurrence of major vascular events. Notably, among DM individuals the observed cardiovascular benefits were largely irrespective of the initial lipid profile, previous vascular events or other baseline characteristics.

\section{Tailored statin therapy}

With statins being used by hundreds of millions people all over the world, it is crucial to learn how to envisage their adverse metabolic effects in selected groups of patients. Physicians should be aware of the fact that there is a gradient for the risk of new-onset DM across different types and doses of statins [31]. Identifying the individuals who would benefit more from smaller doses or use of less diabetogenic compounds could help us to optimize the treatment and reduce the number of people developing DM during hypolipidemic therapy with statins. The moment when statin therapy should be employed in primary prevention also remains controversial and lacks clear definition.

There are no doubts that the use of statins in patients with high cardiovascular risk is fully substantiated. However, it is still uncertain where exactly lies the point beyond which statins' beneficial and protective cardiovascular actions begin to outweigh their small, but apparent, diabetogenic risk. A recent meta-analysis by the aforementioned CTT Collaborators states that even patients with low risk of cardiovascular events gain benefit from cholesterol lowering treatment [44]. In their study, each $1 \mathrm{mmol} / \mathrm{L}$ reduction in LDL-cholesterol concentration produced an absolute reduction in major vascular events of about 11 per 1000 over 5 years. Intriguingly, neither age, sex, baseline LDL-cholesterol, previous vascular disease, nor vascular and all-cause mortality had much impact on the volume of major vascular events risk reduction. Obviously, if patients at low risk of cardiovascular disease become diabetic, their risk of myocardial infarction, stroke, unstable angina or death from cardiovascular causes will skyrocket. The use of hypolipidemic medications with lower potential to negatively interfere with glucose metabolism would sound reasonable in such case.

Based on our findings, pravastatin could be a good match for hyperlipidemic patients assessed to be at low cardiovascular risk. Despite its smaller potential to lower LDL-cholesterol concentration, at the same time it seems to be the least diabetogenic statin currently available on the market. Although a little bit forgotten and marginalized by newer, more powerful and more advertised statins, pravastatin could serve as a valuable alternative, especially for patients with preexisting predispositions for DM. Not to mention the economical aspect of a lower price which most likely could improve the consistency of treatment in all groups of patients. Alternative strategy for such patients could be more cautious dosage, regardless of the prescribed HMG-CoA reductase antagonist. As observed in our meta-analysis, the smaller the daily dose, the lower the incidence of DM.

In people with higher cardiovascular risk and/or very high values of LDL-cholesterol requiring more aggressive therapeutic approach, the treatment with more potent statins, but of worse metabolic profile, cannot be denied. The increased risk of developing DM indisputably has to be considered when introducing rosuvastatin or simvastatin, but cannot be an excluding factor for such therapy. Nevertheless, physicians must be aware of the potential adverse metabolic effects deriving from the therapy consisting of specific statins or higher daily doses. On the other hand, the fact that statins are one of the most efficient known methods of cardiovascular prevention has to be kept in mind at all times. Moreover, it is crucial to remember, that statins cannot be accounted for all the new cases of DM diagnosed during the hypolipidemic therapy. A further analysis from the JUPITER trial clearly shows that the hazard of developing new-onset DM is 
directly connected with the already preexisting DM risk factors [45]. That is why it is so important to put particular emphasis on non-pharmacological methods such as exercises, weight reduction and low fat diet. Additional motivation originating from the physician should not be neglected, as awareness of hypolipidemic therapy may diminish patients' efforts to lead a healthier lifestyle, which in a short period of time can shift them to a higher cardiovascular risk group. These actions, even though very often insufficient, create a chance to decrease baseline LDL-cholesterol concentration and reduce the number of both cardiovascular and DM risk factors. It could enable us to employ a therapy with lower daily doses which, according to our meta-analysis, could confine the number of statin-related DM cases, regardless of the chosen statin. This could be of great importance, particularly with more powerful compounds, but also when larger diabetogenic potential is used.

In our opinion, a regular control of a few commonly available lab tests such as fasting glucose, oral glucose tolerance test or glycated hemoglobin as well as frequent reevaluation of DM risk factors should be recommended in patients chronically using statins. The closer monitoring could enable the early intervention and modification of therapy according to the patient's most recent clinical status. Construction of a simple and clinically applicable scale stratifying patients according to the statin-related DM risk, which would enable physicians to select a specific statin and dose for each patient, could improve the efficacy and safety of HMGCoA reductase antagonists treatment.

\section{Effect of novel non-statin hypolipidemic drugs on new-onset DM}

Routine lipid-lowering therapy is expected to be revolutionized in next years by the widespread use of novel non-statin hypolipidemic drugs, ezetimibe and, particularly, proprotein convertase subtilisin/kexin type 9 inhibitors. Therefore, their impact on glucose metabolism is of major importance.

Based on the results of a randomized, double-blind, placebo-controlled, parallel-group study investigating the effect of ezetimibe vs. placebo on glycated hemoglobin (primary endpoint), glycoalbumin, and fasting plasma glucose concentration in 152 patients with type $2 \mathrm{DM}$ and hypercholesterolemia, Saito et al. found that ezetimibe, an inhibitor of a small intestine cholesterol transporter, does not impair glucose metabolism in this subset of patients [46]. Moreover, two smaller trials indicated that ezetimibe can even improve glucose metabolism in DM patients [47, 48]. Additionally, a substudy of the IMPROVE-IT (IMProved Reduction of Outcomes: Vytorin Efficacy International Trial) trial presented during the recent European Society of Cardiology Congress in London indicated that: 1) diabetics receive greater benefit than nondiabetics with ezetimibe, and 2) ezetimibe does not increase the risk of new-onset DM [49].

Interestingly, recent data from the ODYSSEY Phase 3 program show no significant effect of alirocumab, a proprotein convertase subtilisin/kexin type 9 inhibitor, on glucose metabolism [50]. On the other hand, therapy with second novel proprotein convertase subtilisin/kexin type 9 inhibitor, evolocumab, was associated with a small increase in new-onset DM in patients with impaired fasting glucose [51].

\section{Ongoing trials}

Ongoing trials are expected to shed some light on the issues discussed in this review. However, much more research in this field is needed than the below summarized studies registered in the ClinicalTrials.gov database.

The objective of the randomized, open label, placebo-controlled, parallel-group study Statins on Glucose Homeostasis in Subjects With Impaired Fasting Glucose is to evaluate the effects of rosuvastatin (probably the most diabetogenic statin) and pravastatin (probably protective in terms of the new-onset DM occurrence) on the glucose homeostasis and other biomarkers in 160 subjects with impaired fasting glucose [52]. The study is currently recruiting participants.

The SIPHON (to Evaluate the Safety and Efficacy of Pitavastatin in Patients With Impaired Fasting Glucose and Hyperlipidemia) study is testing the hypothesis on the lack of difference between routine lipid-lowering therapy (pitavastatin $2 \mathrm{mg}$ q.s.) and intensive lipid-lowering therapy (pitavastatin $4 \mathrm{mg}$ q.s.) on glycated hemoglobin concentration [53]. Of note, pitavastatin remains the most potent known statin which is also suggested not to deteriorate glucose metabolism. However, pitavastatin is not approved in Poland yet. The primary study outcome measure is the change of glycated hemoglobin concentration before and after 24 weeks of treatment in a group of 318 patients with impaired fasting glucose and hyperlipidemia. The trial is also currently recruiting participants.

The PPCVD (Primary Prevention of Cardiovascular Disease in Pre-diabetic \& Pre-hypertensive Subjects) aims to determine whether treating pre-diabetic and pre-hypertensive individuals using multiple drugs intervention (anti-hypertensive drugs [i.e., an angiotensin-converting-enzyme inhibitor] plus anti-glycemic drug [i.e., metformin] plus anti-hyperlipidemic drug [a statin]) would lower cardiovascular disease events [54]. The planned enrollment is 8900 patients. The study is not yet open for participants recruitment.

Another registered study not yet recruiting participants is the Statin Strategy Proposal Trial, whose ob- 
jective is to compare the intensity-based statin therapy with attained LDL-cholesterol-based statin protocol in patients with coronary artery disease [55]. The primary outcome measure is the occurrence of major adverse cardiac and cerebrovascular events within 3 years of follow-up in a group of estimated 4400 patients.

\section{Conclusions}

Statins are commonly used in everyday practice both in primary and secondary prevention of cardiovascular events. Statins' status of superstar lipid-lowering drugs with exceptional cardiovascular benefits is undeniable and supported by overwhelming scientific evidence. With special caution regarding the patients with an increased risk of DM, and with the proposed personalized approach, statins should with ease defend their position of a cornerstone of cardiovascular prevention. We cannot forget that statins are not the only diabetogenic drugs that are widely used in cardiology. Beta-blockers, thiazide diuretics and niacin also belong to this group. The adverse actions they exert on glucose metabolism did not exclude them from the routine use due to their favorable cardiovascular effects.

In the guidelines for treating cardiac patients published throughout the recent years the threshold of the optimal LDL-cholesterol concentration has been progressively lowered, following the rule "the lower, the better". It entailed the use of more powerful statins and higher doses, which carries a numerically higher risk of new-onset DM. For that reason it would be advisable to be aware of the fact that in lower risk cases beginning the treatment with smaller daily doses, more careful dosage or choosing less diabetogenic compound could be more beneficial for the patient. There is no doubt that ungrounded use of higher doses of statins increases the probability of DM. This hazard is especially high in patients with already existing conditions like metabolic syndrome, obesity, impaired glucose tolerance or impaired fasting glucose In this subset of patients the use of novel non-statin hypolipidemic drugs can serve as a possible solution. Tailored therapy prepared individually for each patient, after considering all the risk factors as well as potential benefits, may be an answer to our concerns. However, this concept should be further investigated $v$. the one-size-fits-all approach in future trials. Until such studies are completed, we advise to follow five simple rules listed in Figure 2, which may hopefully minimize the risk of new-onset DM in statin-treated patients.

In our opinion, profits derived from statin therapy far exceed the potential harms connected with statin-induced impairments of glucose metabolism. The association between statins and new-onset DM, al-

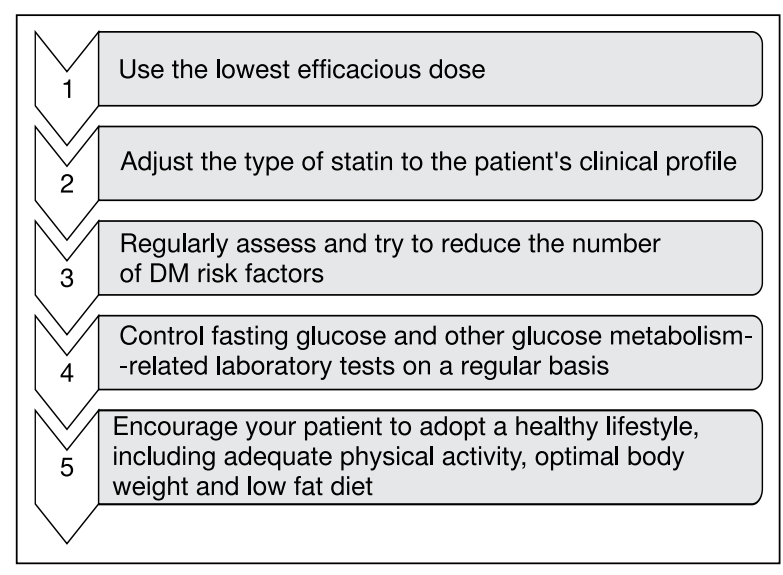

Figure 2. Rules that may help to minimize the risk of new-onset DM in statin-treated patients; DM — diabetes mellitus

though appears to be petite, requires our consideration concerning the widespread use of these compounds and warrants further investigation of the mutual dependencies between DM, statins and cardiovascular risk.

\section{References}

1. Baignent $\mathrm{C}$, Blackwell L, Emberson $\mathrm{J}$ et al. Efficacy and safety of more intensive lowering of LDL cholesterol: a meta-analysis of data from 170 000 participants in 26 randomised trials. Lancet 2010; 376: 1670-1681.

2. Wożakowska-Kapłon B, Filipiak KJ, Mamcarz A et al. Aktualne problemy terapii dyslipidemii w Polsce - II Deklaracja Sopocka. Kardiol Pol 2014; 72: 847-853

3. Krintus M, Kozinski M, Stefanska A et al. Value of C-reactive protein as a risk factor for acute coronary syndrome: a comparison with apolipoprotein concentrations and lipid profile. Mediators Inflamm 2012; 2012: ID 419804.

4. Sypniewska G, Bergmann K, Krintus M, Koziński M, Kubica J. How do apolipoproteins ApoB and ApoA-I perform in patients with acute coronary syndromes. J Med Biochem 2011; 30: 237-243.

5. Jialal I, Stein D, Balis D, Grundy SM, Adams-Huet B, Devaraj S. Effect of hydroxymethylglutaryl coenzyme a reductase inhibitor therapy in high sensitive C-reactive protein levels. Circulation 2001; 103: 1933-1935.

6. Park KW, Hwang KK, Cho HJ et al. Simvastatin enhances endothelial differentiation of peripheral blond mononuclear cells in hypercholesterolemic patients and indu ces pro-angiogenic cytokine IL-8 secretion from monocytes. Clin Chem Acta 2007; 388: 156-166.

7. Bruegel M, Teupser D, Haffner I, Mueller M, Thiery J. Statins reduce macrophage inflammatory protein-1 alpha expression in human activated monocytes. Clin Exp Pharmacol Physiol 2006; 33: 1144-1149.

8. Zeichner S, Mihos CG, Santana O. The pleiotrpic effects and therapeutic potential of the hydroxyl-methyl-glutaryl-CoA reductase inhibitors in malignancies: a comprehensive review. J Cancer Res Ther 2012; 8: 176-183.

9. Sever PS, Dahlöf B, Poulter NR et al.; ASCOT investigators. Prevention of coronary and stroke events with atorvastatin in hypertensive patients who have average or lower-than-average cholesterol concentrations, in the Anglo-Scandinavian Cardiac Outcomes TrialeLipid Lowering Arm (ASCOT-LLA): a multicentre randomised controlled trial. Lancet 2003; 361: 1149-1158.

10. Collins R, Armitage J, Parish S, Sleight P, Peto R; Heart Protection Study Collaborative Group. MRC/BHF Heart Protection Study of cholesterol-lowering with simvastatin in 5963 people with diabetes: a randomised placebo-controlled trial. Lancet 2003; 361: 2005-2016.

11. Freeman DJ, Norrie J, Sattar N et al. Pravastatin and the development of diabetes mellitus: evidence for a protective treatment effect in the West of Scotland Coronary Prevention Study. Circulation 2001; 103: 357-362.

12. Ridker PM, Danielson E, Fonseca FA et al.; JUPITER Study Group. Rosuvastatin to prevent vascular events in men and women with elevated C-reactive protein. N Engl J Med 2008; 359: 2195-2207. 
13. Keech A, Colquhoun D, Best J et al.; LIPID Study Group. Secondary prevention of cardiovascular events with long-term pravastatin in patients with diabetes or impaired fasting glucose: results from the LIPID trial Diabetes Care, 2003: 26: 2713-2721.

14. Kjekshus J, Apetrei E, Barrios V et al.; CORONA Group. Rosuvastatin in older patients with systolic heart failure. N Engl J Med, 2007; 357: 2248-2261.

15. Shepherd J, Blauw GJ, Murphy MB et al.; PROSPER Study Group Pravastatin in elderly individuals at risk of vascular disease (PROSPER): a randomised controlled trial. Lancet 2002; 360: 1623-1630.

16. Nakamura H, Arakawa K, Itakura H et al.; MEGA Study Group.Primary prevention of cardiovascular disease with pravastatin in Japan (MEGA Study): a prospective randomised controlled trial. Lancet 2006; 368: 1155-1163.

17. Downs JR, Clearfield M, Weis $S$ et al. Primary prevention of acute coronary events with lovastatin in men and women with average cholesterol levels: results of AFCAPS/TexCAPS. Air Force/ Texas Coronary Atherosclerosis Prevention Study. JAMA 1998; 279: 1615-1622.

18. Scandinavian Simvastatin Survival Study Group. Randomised trial of cholesterol lowering in 4444 patients with coronary heart disease: the Scandinavian Simvastatin Survival Study (4S). Lancet 1994; 344: 1383-1389.

19. ALLHAT Officers and Coordinators for the ALLHAT Collaborative Research Group. Major outcomes in moderately hypercholesterolemic, hypertensive patients randomized to pravastatin vs usual care: The Antihypertensive and Lipid-Lowering Treatment to Prevent Heart Attack Trial (ALLHAT-LLT). JAMA 2002; 288: 2998-3007.

20. Tavazzi L, Maggioni AP, Marchioli R et al.; GISSI-HF Investigators. Effect of rosuvastatin in patients with chronic heart failure (the GISSI-HF trial): a randomised, double-blind, placebo-controlled trial. Lancet 2008; 372: 1231-1239.

21. GISSI Prevenzione Investigators (Gruppo Italiano per lo Studio Della Sopravvivenza Nell'Infarto Miocardico). Results of the low-dose (20 $\mathrm{mg}$ ) pravastatin GISSI Prevenzione trial in 4271 patients with recent myocardial infarction: do stopped trials contribute to overall knowledge? Ital Heart J 2000; 1: 810-820.

22. Cannon CP, Braunwald E, McCabe $\mathrm{CH}$ et al.; Pravastatin or Atorvastatin Evaluation and Infection Therapye Thrombolysis in Myocardial Infarction 22 Investigators. Intensive versus moderate lipid lowering with statins after acute coronary syndromes. N Engl J Med 2004; 350: 1495-1504.

23. LaRosa JC, Grundy SM, Waters DD et al.; Treating to New Targets (TNT) Investigators. Intensive lipid lowering with atorvastatin in patients with stable coronary disease. N Engl J Med 2005; 352: 1425-1435

24. Pedersen TR, Faergeman O, Kastelein JJ et al.; Incremental Decrease in End Points Through Aggressive Lipid Lowering (IDEAL) Study Group. High-dose atorvastatin vs usual-dose simvastatin for secondary prevention after myocardial infarction: the IDEAL study: a randomized controlled trial. JAMA 2005; 294: 2437-2445.

25. Amarenco P, Bogousslavsky J, Callahan A 3rd et al.; Stroke Prevention by Aggressive Reduction in Cholesterol Levels (SPARCL) Investigators. High-dose atorvastatin after stroke or transient ischemic attack. N Engl J Med 2006; 355: 549-559.

26. Rajpathak SN, Kumbhani DJ, Crandall J, Barzilai N, Alderman M Ridker PM. Statin therapy and risk of developing type 2 diabetes: a meta-analysis. Diabetes Care 2009; 32: 1924-1929.

27. Thongtang N, Otokozawa S, Himbergen TV et al. Effects of maxima atorvastatin and rosuvastatin treatment on markers of glucose homeostasis and inflammation. Am J Cardiol 2011; 107: 387-392.

28. Sattar N, Preiss D, Murray HM et al. Statins and risk of incident diabetes: a collaborative meta-analysis of randomised statin trials. Lance 2010; 375: 735-742.

29. Preiss D, Seshasai SR, Welsh $P$ et al. Risk of incident diabetes with intensive-dose compared with moderate-dose statin therapy: a meta-analysis. JAMA 2011; 305: 2556-2564.

30. FDA expands advice on statin risks. Website of the United States Food and Drug Administration. http://www.fda.gov/ForConsumers/ConsumerUpdates/ucm293330.htm (Accessed 15.11.2015).

31. Navarese EP, Buffon A, Andreotti F et al. Meta-analysis of impact of different types and doses of statins on new-onset diabetes mellitus. Am J Cardiol 2013; 111: 1123-1130.

32. Yada T, Nakata M, Shirishi T, Kakei M. Inhibition by simvastatin, but not pravastatin, of glucose-induced cytosolic $\mathrm{Ca} 2+$ signalling and insulin secretion due to blockade of L-type Ca2 + channels in rat islet beta-cells. Br J Pharmacol 1999; 126: 1205-1213.

33. Mabuchi $H$, Higashikata T, Kawashiri M, et al. Reduction of serum ubiquinol-10 and ubiquinone-10 levels by atorvastatin in hypercholesterolemic patients. J Atheroscler Thromb 2005; 12: 111-119.
34. Nakata M, Uto N, Maruyama I, Yada T. Nitric oxide indu ces apoptosis via $\mathrm{Ca}^{2+}$-dependent processes in the pancreatic beta-cell line MIN6. Cell Struct Funct 1999; 24: 451-455

35. Baker WL, Talati R, White CM, Coleman Cl. Differing effect of statins on insulin sensitivity in non-diabetics: a systemic review and meta-analysis. Diabetes Res Clin Pract 2010; 87: 98-107.

36. Diez JJ, Iglesias P. The role of the novel adipocyte-derived hormone adiponectin in human disease. Eur J Endocrinol 2003: 148: 293-300.

37. Koh KK, Quon MJ, Han SH et al. Simvastatin improves flow-mediated dilation but reduces adiponectin levels and insulin sensitivity in hypercholesterolemic patients. Diabetes Care 2008; 31: 776-782.

38. Koh KK, Quon MJ, Han SH et al. Differential metabolic effects of pravastatin and simvastatin in hypercholesterolemic patients. Atherosclerosis 2009; 204: 483-490.

39. Bhandari S, Gupta P, Quinn P et al. Pleiotropic effects of statins in hypercholesterolaemia: a prospective observational study using a lipoproteomic based approach. Lancet 2015; 385: 21.

40. Nakata M, Nagasaka S, Kusaka I, Matsuoka H, Ishibashi S, Yada T. Effects of statins on the adipocytte maturation and expression of glucose transporter 4 (SLC2A4): implications in glycaemic control. Diabetologia 2006; 49: 1881-1892.

41. Chamberlain LH. Inhibition of isoprenoid biosynthesis causes insulin resistance in 3T3-L1 adipocytes. PEBS Lett 2001; 507: 357-361.

42. Swerdlow DI, Preiss D, Kuchenbaecker KB et al. HMG-coenzyme A reductase inhibition, type 2 diabetes, and bodyweight: evidence from genetic analysis and randomised trials. Lancet 2015; 385: 351-361.

43. Cholesterol Treatment Trialists' (CTT) Collaborators, Kearney PM, Blackwell L, Collins R et al. Efficacy of cholesterol-lowering therapy in 18,686 people with diabetes in 14 randomised trials of statins: a meta-analysis. Lancet 2008; 371: 117-125.

44. Cholesterol Treatment Trialists' (CTT) Collaborators, Mihaylova B, Emberson J, Blackwell $L$ et al. The effects of lowering LDL cholesterol with statin therapy in people at low risk of vascular disease: meta-analysis of individual data from 27 randomised trials. Lancet 2012; 380: 581-590.

45. Ridker PM, Pradhan A, MacFadyen JG, Libby P, Glynn RJ. Cardiovascular benefits and diabetes risks of statin therapy in primary prevention: an analysis from the JUPITER trial. Lancet 2012; 380: 565-571.

46. Saito I, Azuma K, Kakikawa T, Oshima N, Hanson ME, Tershakovec AM. A randomized, double-blind, placebo-controlled study of the effect of ezetimibe on glucose metabolism in subjects with type 2 diabetes mellitus and hypercholesterolemia. Lipids Health Dis 2015; 14: 40.

47. Nozue T, Michishita I, Mizuguchi I. Effects of ezetimibe on glucose metabolism in patients with type 2 diabetes: A 12-week, open-label, uncontrolled, pilot study. Curr Ther Res Clin Exp 2010; 71: 252-258.

48. Tsunoda T, Nozue T, Yamada M, Mizuguchi I, Sasaki M, Michishita I. Effects of ezetimibe on atherogenic lipoproteins and glucose metabolism in patients with diabetes and glucose intolerance. Diabetes Res Clin Pract 2013; 100: 46-52.

49. Blazing MA. Incidence of new-onset diabetes in the IMPROVE-IT trial: does adding ezetimibe to simvastatin increase risk compared to simvastatin alone? Presented at: the European Society of Cardiology Congress; September 1, 2015; London, England.

50. Colhoun HM, Ginsberg HN, Robinson JG et al. Alirocumab effect on glycemic measures in patients without diabetes at baseline. Circulation 2015; 132: A16863

51. Risk evaluation and mitigation strategy review on evolocumab. Website of the United States Food and Drug Administration. http://www.accessdata.fda.gov/drugsatfda_docs/nda/2015/125522Orig1s000RiskR.pdf (Accessed 15.11.2015)

52. Statins on Glucose Homeostasis in Subjects With Impaired Fasting Glucose study. Website of the ClinicalTrials.gov. https://clinicaltrials. gov/ct2/show/NCT01816997?term =NCT01816997\&rank=1. (Accessed 15.11.2015)

53. To Evaluate the Safety and Efficacy of Pitavastatin in Patients With IFG and Hyperlipidemia (SIPHON). Website of the ClinicalTrials. gov. https://clinicaltrials.gov/ct2/show/NCT02056847? term=NCT02056847\&rank=1. (Accessed 15.11.2015).

54. Primary Prevention of Cardiovascular Disease (CVD) in Pre-diabetic \& Pre-hypertensive Subjects (PPCVD). Website of the ClinicalTrials. gov. https://clinicaltrials.gov/ct2/show/NCT013 64675?term=NCT01364675\&rank=1. (Accessed 15.11.2015).

55. Comparing the Intensity-based Statin Therapy With Attained Low-density Lipoprotein Cholesterol Based Statin Therapy in Patients With Coronary Artery Disease: Statin Strategy Proposal Trial. Website of the ClinicalTrials.gov. https://clinicaltrials.gov/ct2/show/NCT02579499?term $=$ NCT0257949 9\&rank=1. (Accessed 15.11.2015). 